\title{
6-Shogaol, an active compound of ginger, protects dopaminergic neurons in Parkinson's disease models via anti-neuroinflammation
}

\author{
Gunhyuk PARK ${ }^{1}$, Hyo Geun $\mathrm{KIM}^{2}$, Mi Sun $\mathrm{JU}^{2}$, Sang Keun $\mathrm{HA}^{3}$, Yongkon PARK ${ }^{3}$, Sun Yeou $\mathrm{KIM}^{4}$, Myung Sook $\mathrm{OH}^{1,2, *}$ \\ ${ }^{1}$ Department of Life and Nanopharmaceutical Science, Kyung Hee University, Seoul 130-701, Republic of Korea; ${ }^{2}$ Department of \\ Oriental Pharmaceutical Science, College of Pharmacy and Kyung Hee East-West Pharmaceutical Research Institute, Kyung Hee Uni- \\ versity, Seoul 130-701, Republic of Korea; ${ }^{3}$ Functional Materials Research Group, Korea Food Research Institute, Gyeonggi 463-746, \\ Republic of Korea; ${ }^{4}$ College of Pharmacy, Gachon University, Incheon 406-799, Republic of Korea
}

Aim: 6-Shogaol [1-(4-hydroxy-methoxyphenyl)-4-decen-one], a pungent compound isolated from ginger, has shown various neurobiological and anti-inflammatory effects. The aim of this study was to examine the effects of 6-shogaol on neuroinflammatory-induced damage of dopaminergic (DA) neurons in Parkinson's disease (PD) models.

Methods: Cultured rat mesencephalic cells were treated with 6 -shogaol $(0.001$ and $0.01 \mu \mathrm{mol} / \mathrm{L})$ for $1 \mathrm{~h}$, then with $\mathrm{MPP}{ }^{+}(10 \mu \mathrm{mol} / \mathrm{L})$ for another $23 \mathrm{~h}$. The levels of TNF- $\alpha$ and NO in medium were analyzed spectrophotometrically. C57/BL mice were administered 6-shogaol $\left(10 \mathrm{mg} \cdot \mathrm{kg}^{-1} \cdot \mathrm{d}^{-1}, \mathrm{po}\right)$ for $3 \mathrm{~d}$, and then MPTP $(30 \mathrm{mg} / \mathrm{kg}$, ip) for $5 \mathrm{~d}$. Seven days after the last MPTP injection, behavioral testings were performed. The levels of tyrosine hydroxylase (TH) and macrophage antigen (MAC)-1 were determined with immunohistochemistry. The expression of iNOS and COX-2 was measured using RT PCR.

Results: In $\mathrm{MPP}^{+}$-treated rat mesencephalic cultures, 6-shogaol significantly increased the number of TH-IR neurons and suppressed TNF- $\alpha$ and NO levels. In C57/BL mice, treatment with 6-shogaol reversed MPTP-induced changes in motor coordination and bradykinesia. Furthermore, 6-shogaol reversed MPTP-induced reductions in TH-positive cell number in the substantia nigra pars compacta (SNpc) and TH-IR fiber intensity in stratum (ST). Moreover, 6-shogaol significantly inhibited the MPTP-induced microglial activation and increases in the levels of TNF- $\alpha$, NO, iNOS, and COX-2 in both SNpc and ST.

Conclusion: 6-Shogaol exerts neuroprotective effects on DA neurons in in vitro and in vivo PD models.

Keywords: 6-shogaol; ginger; dopaminergic neurons; substantia nigra; stratum; Parkinson's disease; neuroinflammation; microglia; 1-methyl-4-phenyl-1,2,3,6-tetrahydropyridine (MPTP)

Acta Pharmacologica Sinica (2013) 34: 1131-1139; doi: 10.1038/aps.2013.57; published online 1 Jul 2013

\section{Introduction}

Microglial cells, resident immune cells in the brain, play important roles in the onset of neuroinflammatory responses in the central nervous system and are readily activated during most neuropathological conditions ${ }^{[1]}$. Microglial activation involves cell proliferation and migration to the injury site, increased expression of immunomodulators, and ultimately cell transformation into phagocytes that are capable of clearing damaged cells and debris ${ }^{[2]}$. Activated microglia can also promote neuronal injury through the release of proinflammatory and cytotoxic factors, including tumor necrosis factor (TNF)- $a$, nitric oxide (NO), inducible nitric oxide synthase (iNOS), and

\footnotetext{
* To whom correspondence should be addressed.

E-mail msohok@khu.ac.kr

Received 2013-02-15 Accepted 2013-04-12
}

cyclooxygenase-2 (COX-2) ${ }^{[3]}$. Chronic microglial activation has been implicated in the neuronal destruction associated with neurodegenerative diseases such as Alzheimer's disease and Parkinson's disease (PD) ${ }^{[2,3]}$. Thus, the activation of regulatory mechanisms is essential to avoid the escalation of central nervous system inflammatory processes ${ }^{[4]}$. This may be possible through the identification of therapeutic agents that target over-activated microglia and the determination of their underlying anti-neuroinflammatory mechanisms.

Previous studies have shown that 6-shogaol [1-(4-hydroxymethoxyphenyl)-4-decen-one], a pungent compound isolated from ginger, has various neurobiological and anti-inflammatory effects(Figure 1) ${ }^{[5-7]}$. It reduced cell death and restored motor function in rat spinal cord injury, attenuated dopamine depression in response to 6-hydroxydopamine, and prevented the proliferation of glial cells via an increase of brain- 


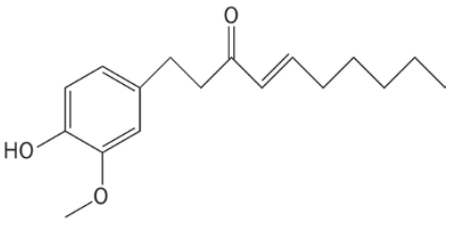

Figure 1. Chemical structure of 6-shogaol.

derived neurotrophic factor in lipopolysaccharide-treated astrocytes ${ }^{[8-10]}$. This compound has been shown to protect hippocampal neurons via an increase in choline acetyltransferase and the choline transporter ${ }^{[11]}$. In in vitro and in vivo inflammatory models, 6-shogaol protected microglia against lipopolysaccharide-induced toxicity by inhibiting the production of $\mathrm{NO}$, prostaglandin $\mathrm{E}$, and proinflammatory cytokines such as TNF- $\alpha$ and interleukin- $1 \beta$ via the downregulation of COX-2, iNOS, p38 mitogen-activated protein kinase, and nuclear factor kappa B (NF-kB) expression ${ }^{[12]}$. Moreover, 6-shogaol exhibited neuroprotective effects via the inhibition of microglia in an in vivo model of transient global ischemia ${ }^{[12]}$. However, no studies have investigated its effects on neuroinflammatory damage in dopaminergic neurons using a PD model.

Therefore, this study examined the protective effects of 6-shogaol against 1-methyl-4-phenylpyridinium $\left(\mathrm{MPP}^{+}\right)$- or 1-methyl-4-phenyl-1,2,3,6-tetrahydropyridine (MPTP)-induced movement impairment and dopaminergic neuron death using behavioral tests and immunohistochemical analysis of primary mesencephalic cells in a PD model. The ability of 6-shogaol to repress microglial activation and its possible underlying mechanisms were investigated by assessing the levels of TNF-a, NO, iNOS, and COX-2.

\section{Materials and methods Materials}

6-Shogaol was purchased from Wako Pure Chemical (Osaka, Japan). Fetal bovine serum (FBS), penicillin, and streptomycin were purchased from Hyclone Laboratories Inc(Logan, UT, USA). Minimum essential medium (MEM) were purchased from Gibco Industries Inc (Auckland, New Zealand). $\mathrm{MPP}^{+}$, MPTP, poly-L-lysine (PLL), glucose, glutamine, sodium nitrite $\left(\mathrm{NaNO}_{2}\right)$, phosphoric acid, naphthylethylene diamide, sulfanilamide, paraformaldehyde (PFA), 3,3'-diaminobenzidine (DAB), phosphate buffer (PB), phosphate buffered saline (PBS), glycerin, ethylene glycol, Triton X-100, dimethyl sulfoxide (DMSO) were purchased from Sigma-Aldrich (St Louis, MO, USA). Rabbit anti-tyrosine hydroxylase (TH) affinity-purified polyclonal antibody and rat anti-macrophage antigen (MAC)-1 monoclonal antibody were obtained from Chemicon International Inc (Temecula, CA, USA). Biotinylated anti-rabbit and rat antibodies, normal goat serum, and avidin-biotin peroxidase complex $(\mathrm{ABC})$ standard kit were purchased from Vector Laboratories (Burlingame, CA, USA). Rat TNF-a assay kit, Trizol reagent, and moloney murine leukemia virus (MMLV) reverse transcriptase were purchased from Invitrogen Life Technologies (Carlsbad, CA, USA) and
QuantiTect SYBR Green PCR kit was purchased from Qiagen (Valencia, CA, USA). Zoletil was purchased from Virbac (Carros, France). iNOS, COX-2, and glyceraldehyde-3-phosphate dehydrogenase (GAPDH) in oligonucleotide primers were purchased from Bioneer (Daejeon, Korea). The other reagents used were of guaranteed or analytical grade.

\section{Primary cultures of rat mesencephalic cells}

The cell cultures were prepared from the ventral mesencephalons of 14-day embryos of timed pregnant Sprague-Dawley rats (Orient Bio, Osan, Korea). Briefly, after the mesencephalons were dissected, collected, and dissociated, the cells were seeded onto PLL pre-coated cover slips in 24-well plates at a density of $1.5 \times 10^{5}$ cells/well in MEM supplemented with 6.0 $\mathrm{g} / \mathrm{L}$ glucose, $2 \mathrm{mmol} / \mathrm{L}$ glutamine and 10\% FBS. Cultures were maintained in a water-saturated atmosphere of $5 \% \mathrm{CO}_{2}$ at $37^{\circ} \mathrm{C}$. Seven-day-old cultures were used for treatment. The composition of the cells at the time of treatment was $45 \%$ astrocytes, 5\% microglia and 50\% neurons with $6 \%$ of the neurons being dopaminergic neurons. The cells were treated with 6-shogaol $(0.001$ and $0.01 \mu \mathrm{mol} / \mathrm{L})$ for $1 \mathrm{~h}$ and then with $10 \mu \mathrm{mol} / \mathrm{L} \mathrm{MPP}^{+}$for an additional $23 \mathrm{~h}$. After culture supernatants were collected separately, the treated cells were fixed with $4 \%$ PFA for $30 \mathrm{~min}$ at room temperature for immunohistochemistry (IHC).

\section{Measurement of extracellular NO and TNF- $\alpha$}

The accumulated level of NO in culture supernatants was measured using the colorimetric reaction with the Griess reagent for $10 \mathrm{~min}$ at room temperature in a dark. Absorbance was measured at a wavelength of $570 \mathrm{~nm}$ using a spectrophotometer (Versamax microplate reader; Molecular Devices, Sunnyvale, CA, USA) and concentrations of NO was determined by $\mathrm{NaNO}_{2}$ standard curve. TNF-a assay was performed according to the each manufacturer's protocol using culture supernatants. Briefly, levels of TNF-a were measured using spectrophotometer at $450 \mathrm{~nm}$, and then data were expressed as standard curves of TNF- $\mathrm{a}(\mathrm{pg} / \mathrm{mL})$.

\section{Animals and treatment}

Male C57BL/ 6 mice at 7 weeks of age, weighing 22-23 g, were purchased from the Samtako Co, Ltd (Osan, Korea). Animals were housed 10 per cage, were allowed access to water and food ad libitum, and were maintained at a constant temperature $\left(23 \pm 1^{\circ} \mathrm{C}\right)$ and humidity $(60 \% \pm 10 \%)$ under a 12-h light/ dark cycle (light on 07:30-19:30). Animal treatment and maintenance were carried out in accordance with the Principle of Laboratory Animal Care (NIH publication No $85-23$, revised 1985) and with the Animal Care and Use Guidelines of Kyung Hee University, Korea. Animals were assigned into three groups; (1) control group $(n=10)$, (2) MPTP group $(n=10)$, and (3) MPTP+6-shogaol group $(n=10)$. MPTP $\left(30 \mathrm{mg} \cdot \mathrm{kg}^{-1} \cdot \mathrm{d}^{-1}, \mathrm{ip}\right)$ dissolved in normal saline were administered for $5 \mathrm{~d}$. Also, following the optimal dosage conditions used in previously published study, 6-shogaol $\left(10 \mathrm{mg} \cdot \mathrm{kg}^{-1} \cdot \mathrm{d}^{-1}, \mathrm{po}\right)$ dissolved in $10 \%$ DMSO was administered for the last $3 \mathrm{~d}$ of MPTP treat- 
ment. MPTP was injected two hours after 6-shogaol treatment and equal volumes of vehicles were given to the control and MPTP groups.

\section{Behavioral test}

The pole test is a useful method in mice PD model to measure bradykinesia. We performed the pole test on the seventh day after the last MPTP injection. The mice were held on the top of the pole (diameter $8 \mathrm{~mm}$, height $55 \mathrm{~cm}$, with rough surface). The time that mice needed to turn down completely and climb down and four feet reached the floor was recorded. Each trial had the cut-off limit of $30 \mathrm{~s}$.

\section{Brain tissue preparation}

The mice were anesthetized with Zoletil $50^{\circledR}(10 \mathrm{mg} / \mathrm{kg}, \mathrm{im})$ and transcardially perfused with $100 \mathrm{mmol} / \mathrm{L} \mathrm{PB}(\mathrm{pH} 7.4)$ followed by ice-cold $4 \%$ PFA. Their brains were isolated and post-fixed in $\mathrm{PB}(50 \mathrm{mmol} / \mathrm{L}, \mathrm{pH} 7.4)$ containing 4\% PFA overnight, immersed in a solution containing $30 \%$ sucrose in 50 $\mathrm{mmol} / \mathrm{L}$ PBS and stored at $4{ }^{\circ} \mathrm{C}$ until sectioning. The frozen brains were coronally sectioned on a cryostat at $30 \mu \mathrm{m}$ and then stored in a storage solution at $4^{\circ} \mathrm{C}$.

\section{Immunohistochemistry}

Free floating sections or mesencephalic cells on cover slips were incubated in PBS containing anti-TH antibody (1:2000 dilution) or anti-MAC-1 antibody (1:1000 dilution), $0.3 \%$ Triton $\mathrm{X}-100,0.5 \mathrm{mg} / \mathrm{mL}$ bovine serum albumin at $4{ }^{\circ} \mathrm{C}$ for $24 \mathrm{~h}$. They were then incubated for $90 \mathrm{~min}$ with biotinylated secondary antibody (1:200 dilution), followed by incubation in $\mathrm{ABC}$ solution for $1 \mathrm{~h}$ at room temperature. The color was developed with DAB. After every incubation step, they were washed three times with PBS. The mesencephalic cells on cover slips were mounted on gelatin-coated slides and air dried. The free floating brain tissue sections were mounted on gelatin-coated slides, dehydrated with an ascending alcohol, cleared with xylene, and cover slipped using Histomount ${ }^{\mathrm{TM}}$ (National Diagnostics, Atlanta, GA, USA). Quantifications of the effects in primary mesencephalic dopaminergic cells were performed by counting the TH-immunoreactive (TH-IR) cells in entire areas of five wells of each group at $\times 100$ magnification and by measuring TH-IR in ten representative areas per well in five wells of each group at $\times 200$ magnification. Quantification of effect in brain tissue sections were performed by counting the numbers of the TH-IR cells in the substantia nigra pars compacta (SNpc) and measuring the optical intensity of TH-IR fibers in striatum (ST) at $\times 100$ and $\times 40$ magnifications, respectively, using Stereoinvestigator software (MBF Bioscience Inc, Williston, VT, USA) and then presented as a percent of the control group values. The images were photographed with a research microscope (BX51T-32F01; Olympus Corporation, Tokyo, Japan)

\section{Western blot analysis}

For the detection of TH, MAC-1, iNOS, and COX-2 proteins, the SNpc or ST tissues were lysed. The lysates were separated by $10 \%$ SDS-PAGE, and were then transferred to a membrane. The membranes were incubated with 5\% skim milk in TBST for $1 \mathrm{~h}$. Then they were incubated with rabbit anti-TH, MAC1 , iNOS (1:500 dilutions), mouse anti- $\beta$-actin (1:3000 dilutions), and goat anti-COX-2 (1:250 dilutions) primary antibody overnight at $4{ }^{\circ} \mathrm{C}$, followed by incubation with HRP-conjugated anti-mouse and rabbit IgG for $1 \mathrm{~h}$, respectively. Immunoreactive bands were detected using an ECL detection kit and a LAS-4000 mini system (Fujifilm Corporation, Tokyo, Japan) was used for visualization. The intensities of the bands were normalized to the $\beta$-actin band intensity using Multi Gauge software (Fujifilm Corporation, Tokyo, Japan).

Reverse transcriptase (RT) real-time polymerase chain reaction (PCR)

Total RNA was isolated using Trizol reagent according to the manufacturer's protocol. RNA (1 $\mu \mathrm{g})$ was reverse-transcribed in $20 \mu \mathrm{L}$ reaction mixture using MMLV reverse transcriptase. For real-time PCR, cDNAs $(0.2 \mu \mathrm{L})$ were amplified using a QuantiTect SYBR Green PCR kit in a Rotor-Gene 3000 (Corbett Research, Sydney, Australia). The cycle profile used was an initial denaturation at $95^{\circ} \mathrm{C}$ for $10 \mathrm{~min}$ followed by 40 cycles of at $95^{\circ} \mathrm{C}$ for $15 \mathrm{~s}, 60^{\circ} \mathrm{C}$ for $10 \mathrm{~s}$, and $72^{\circ} \mathrm{C}$ for $20 \mathrm{~s}$. As a control for normalizing sample loading, PCR amplification of the housekeeping gene GAPDH was included for each sample in each run. Nucleotide sequences of the primers were based on published cDNA sequences of mouse iNOS (iNOS forward, 5'-GGCAACATCAGGTCGGCCATTACTG-3'; iNOS reverse, 5'-GGAACCACTCGTACTTGGGATGCTC-3'), COX-2 (COX-2 forward, 5'-CCGTGGTGAATGTATGAGCA-3'; COX-2 reverse, 5'-GATTGACAGCCCACCAACTT-3'), and GAPDH (GAPDH forward, 5'-TGTCAACGGATTTGGCCGTATTGGC-3'; GAPDH reverse, 5'-GAAGACGCCAGTAGACTCCACGAC- $\left.3^{\prime}\right)$. Fluorescence measurements were obtained online and were analyzed with Rotor-Gene 3000 software (ver 6.0; Corbett Research). Gene expression was quantitated using the comparative threshold cycle method as described.

\section{Statistical analysis}

All statistical parameters were calculated using GraphPad Prism 4.0 software (GraphPad Software Inc, San Diego, CA, USA). Values were expressed as the mean \pm standard error of the mean (SEM). The results were analyzed by one-way analysis of variance. Differences with a $P$-value less than 0.05 were considered statistically significant.

\section{Results}

Effect of 6-shogaol against MPP ${ }^{+}$-induced neurotoxicity in primary rat mesencephalic culture

To assess the protective effects of 6-shogaol on dopaminergic neurons, we counted TH-IR neurons. Treatment with $\mathrm{MPP}^{+}$reduced the number of TH-IR neurons to $63.14 \% \pm 3.61 \%$ compared to the control group. However, 6-shogaol treatment at 0.001 and $0.01 \mu \mathrm{mol} / \mathrm{L}$ prevented dopaminergic cell loss induced by $\mathrm{MPP}^{+}$toxicity, showing $84.53 \% \pm 1.21 \%$ and $98.37 \% \pm 10.27 \%$ of the control group (Figure 2 ). 
Effect of 6-shogaol on MPP ${ }^{+}$-induced production of neuroinflammatory factors in primary mesencephalic culture

To investigate the effect of 6-shogaol on inflammatory response induced by $\mathrm{MPP}^{+}$toxicity, we examined levels of $\mathrm{NO}$ and TNF- $\alpha$ using cell supernatants. In this study, $\mathrm{MPP}^{+}-$ treated group showed significant increase of NO and TNF- $\alpha$ levels to $122.44 \% \pm 0.04 \%$ and $170.20 \% \pm 25.43 \%$ compared to the control group, respectively. In contrast, treatment with 6-shogaol at 0.001 and $0.01 \mu \mathrm{mol} / \mathrm{L}$ dose-dependently suppressed the increased levels, from $78.95 \% \pm 6.05 \%$ to $73.65 \% \pm 8.60 \%$ and from $56.05 \% \pm 11.65 \%$ to $14.65 \% \pm 9.19 \%$ compared to the control group, respectively (Figure 3).

\section{Effects of 6-shogaol on MPTP-induced movement impairment of} mice

To examine the effects of 6-shogaol against MPTP neurotoxicity, we measured movement impairment using the pole tests. In this study, T-turn and T-LA were significantly prolonged to $164.44 \% \pm 9.95 \%$ and $127.86 \% \pm 3.51 \%$ compared with the control group, respectively. However, T-turn and T-LA were significantly shortened in the MPTP+6-shogaol group to $108.02 \% \pm 5.50 \%$ and $105.01 \% \pm 3.51 \%$ compared with the control at dose $10 \mathrm{mg} / \mathrm{kg}$ (Figure 4).

Effects of 6-shogaol on MPTP-induced dopaminergic neuronal loss in the SNpc and ST

To confirm the effects of 6-shogaol on dopaminergic damage, we performed TH-IHC in the SNpc and ST. In the MPTP-only treated mice, the number of TH-positive cells in the SNpc and the optical intensity in the ST were decreased to $76.77 \% \pm 3.42 \%$ and $74.72 \% \pm 2.14 \%$ compared with the control group, respec-

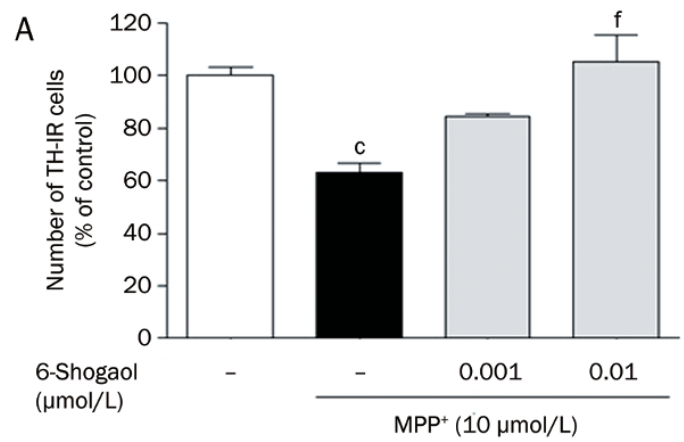

B
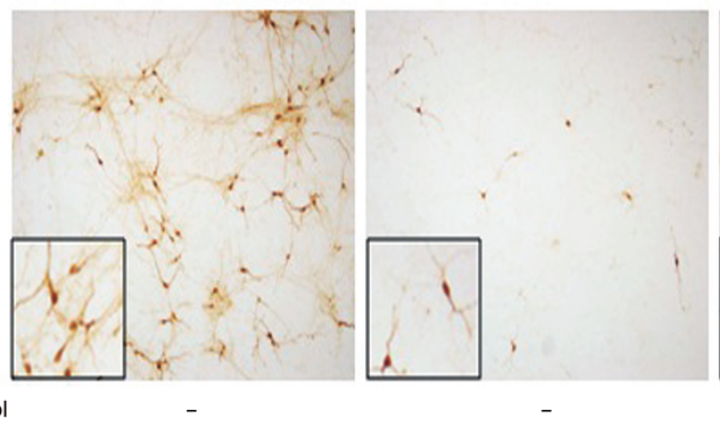

6-Shogaol $(\mu \mathrm{mol} / \mathrm{L})$
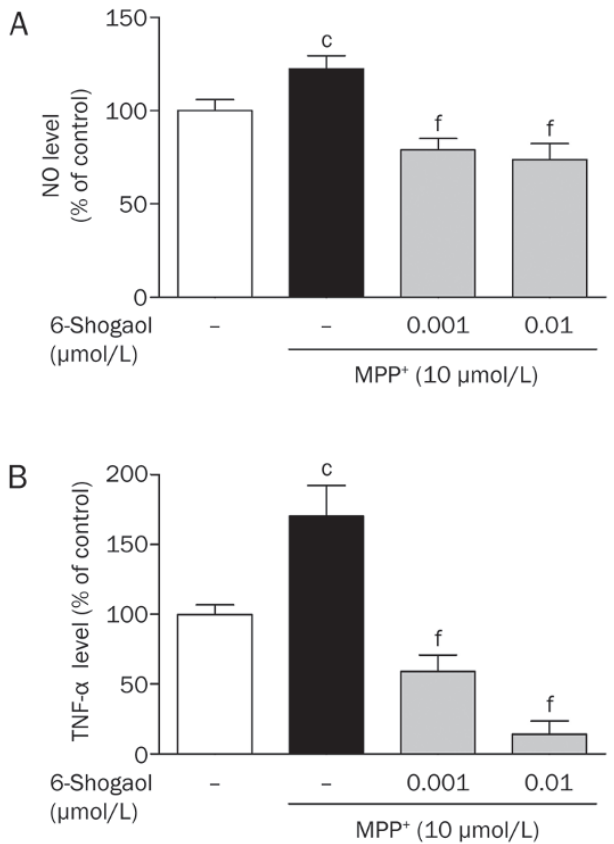

Figure 3. Inhibitory effects of 6-shogaol on $\mathrm{MPP}^{+}$-induced neuroinflammatory factors in primary mesencephalic cells. After the cells were treated with 6-shogaol and/or $\mathrm{MPP}^{+}$, the culture supernatant was used to measure NO (A) and TNF- $\alpha$ levels (B). Values are indicated as mean \pm SEM. ${ }^{\mathrm{c}} P<0.01$ compared with the control group. ${ }^{\mathrm{f}} P<0.01$ compared with the $\mathrm{MPP}^{+}$-only treated group.

tively. However, these values are significantly increased by 6 -shogaol $10 \mathrm{mg} \cdot \mathrm{kg}^{-1} \cdot \mathrm{d}^{-1}$ treatment as $90.42 \% \pm 1.81 \%$ and $88.78 \% \pm 1.16 \%$ compared with the control group (Figure 5).

Figure 2. The protective effects of 6-shogaol against MPP ${ }^{+}$-induced neurotoxicity in primary mesencephalic cells. Cells were treated with 6-shogaol for $1 \mathrm{~h}$ and then exposed to $\mathrm{MPP}^{+}$for a further $23 \mathrm{~h}$. After the cells were fixed and stained, the number of tyrosine hydroxylase (TH)immunoreactive neurons was measured $(A)$. Representative images of experiments are shown (B). Scale bar $=50 \mu \mathrm{m}$. Values are indicated as mean \pm SEM. ${ }^{c} P<0.01$ compared with the control group. ${ }^{f} P<0.01$ compared with the $\mathrm{MPP}^{+}$-only treated group.

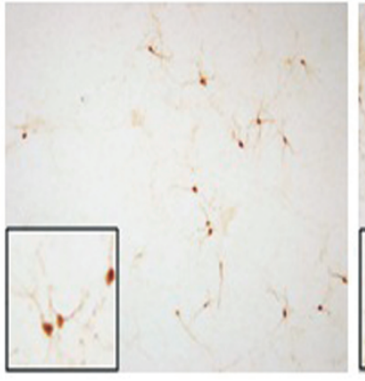

0.001

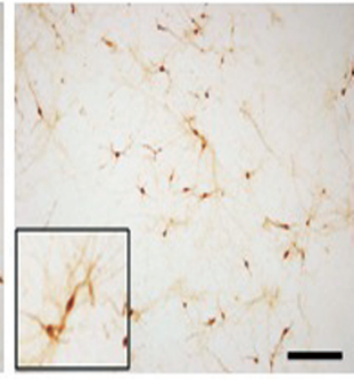

0.01

$\mathrm{MPP}^{+}(10 \mu \mathrm{mol} / \mathrm{L})$ 
A

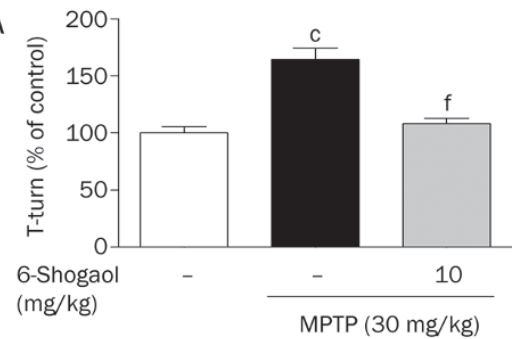

B

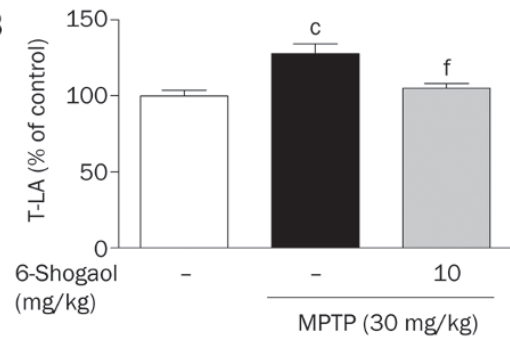

Figure 4. Inhibitory effects of 6-shogaol on MPTP-induced movement impairment in a mouse PD model. Saline or 6-shogaol $(10 \mathrm{mg} / \mathrm{kg}$; po) was administered to mice once per day for $3 \mathrm{~d}$, and then MPTP (30 mg/kg) was injected intraperitoneally for $5 \mathrm{~d}$. Seven days after the last MPTP injection, the time required for the mouse to turn completely downward $(A$, T-turn) and the time required to arrive at the floor (B, T-LA) were recorded, using a cut-off limit of $60 \mathrm{~s}$. Values are indicated as mean \pm SEM. ${ }^{\mathrm{C}} P<0.01$ compared with the control group. ${ }^{f} P<0.01$ compared with the MPTP-only treated group.

Effects of 6-shogaol on MPTP-induced microglial activation and iNOS and COX-2 expression in mice

To examine whether 6-shogaol affects microglial activation, we performed IHC using MAC-1 antibody. In this study, MPTP-treated group showed increase of activated microglia in the SNpc and ST to $465.66 \% \pm 23.38 \%$ and $370.26 \% \pm 12.29 \%$ of MAC-1-IR optical intensity compared to the control group, respectively. However, 6-shogaol inhibited the MPTPinduced microglial activation in the SNpc and ST, showing $342.95 \% \pm 21.67 \%$ and $284.22 \% \pm 19.16 \%$ of MAC-1-IR optical intensity, respectively, compared to the MPTP-treated group (Figure 6). In addition, we measured iNOS and COX-2 expression levels which are released by inflammatory signal using real time RT-PCR and Western blot analysis. MPTP-treated group had significantly increased level of iNOS by $29.30 \% \pm 5.30 \%$ and $36.20 \% \pm 6.10 \%$ of the control and $1237.32 \% \pm 78.43 \%$ and $770.66 \% \pm 38.40 \%$ of the control in the $\mathrm{SNpc}$ and the ST, respectively. In contrast, treatment with 6-shogaol suppressed it to $12.90 \% \pm 4.30 \%$ and $13.40 \% \pm 5.50 \%$ of the control and $799.71 \% \pm 51.14 \%$ and $695.87 \% \pm 34.84 \%$ of the control, respectively. Similar to the result of iNOS, COX-2 is excessively expressed by MPTP toxicity, by $34.90 \% \pm 5.50 \%$ and $40.70 \% \pm 5.90 \%$ of the control and $406.63 \% \pm 22.72 \%$ and $134.10 \% \pm 7.49 \%$ of the control in the SNpc and the ST whereas it was suppressed by treatment of 6-shogaol, showing $15.50 \% \pm 3.90 \%$ and $24.10 \% \pm 4.40 \%$ of the control and $245.28 \% \pm 17.63 \%$ and $109.54 \% \pm 7.87 \%$ of the control, respec- tively (Figure 7).

\section{Discussion}

The present study demonstrated that 6-shogaol protects against MPTP- or $\mathrm{MPP}^{+}$-induced movement impairment and neuronal damage by regulating microglial activation and downstream proinflammatory factors in a mouse model of PD.

To examine the protective effects of 6-shogaol against $\mathrm{MPP}^{+}$ or MPTP neurotoxicity, impairments in movement and alterations of dopaminergic neurons were examined. MPTP, a neurotoxin, is routinely used to induce PD-like neurodegeneration in experimental animal models ${ }^{[13]}$. In the brain, MPTP is converted to the active metabolite $\mathrm{MPP}^{+}$by monoamine oxidase $\mathrm{B}$ within non-dopaminergic cells, after which $\mathrm{MPP}^{+}$enters dopaminergic neurons via the dopamine transporter ${ }^{[14]}$. When $\mathrm{MPP}^{+}$accumulates in dopaminergic neurons in the SNpc, it inhibits complex I of the mitochondrial electron transport chain, ultimately leading to toxic injury and dopaminergic cell degeneration $^{[14]}$. The loss of dopaminergic neurons is associated with the onset of motor deficits in which there is a direct relationship between the extent of dopamine loss and motor dysfunction ${ }^{[15]}$. The present study showed that 6-shogaol treatment significantly improved MPTP-induced motor deficits and bradykinesia in pole test. Moreover, histological analyses illustrated that 6-shogaol treatment significantly protected dopaminergic neurons from this toxicity and resulted in an increased number of TH-IR cells and the preservation of dopaminergic neuronal morphology in rat primary mesencephalic cells and in mice. Previous studies reported that 6-shogaol protected neuronal cell against neurotoxicity-induced damage $^{[12]}$.

To investigate the protective effects of 6-shogaol against $\mathrm{MPP}^{+}$- or MPTP-induced activation of microglia and downstream proinflammatory factors, the activation of MAC-1 and levels of TNF- $a$, NO, iNOS, and COX-2 were examined. The specific actions of $\mathrm{MPP}^{+}$and MPTP in glial and microglial activation associated with inflammatory mediator production remain ambiguous ${ }^{[16]}$. In the brain, MPTP induces reactive gliosis, the cellular manifestation of neuroinflammation, as a common response to neuronal injury ${ }^{[17]}$. Reactive gliosis is characterized by the activation of either microglia or astroglia, both of which are able to respond to injury and participate in pathological events ${ }^{[18]}$. Activated microglial cells contribute to neuronal degeneration because they produce a broad array of neurotoxic molecules, including proinflammatory cytokines, chemokines, and prostaglandins, as well as a large amount of reactive oxygen and nitrogen species ${ }^{[18-20]}$. The major intracellular events that may be associated with microglial activation are the induction of TNF-a and NO and the elevated expression of COX-2 and iNOS ${ }^{[21]}$. In particular, TNF-a may have a direct damaging effect on dopaminergic neurons by activating an intracellular death pathway coupled with TNF receptor-1, which is expressed on the cell surface of dopamine neurons ${ }^{[3,22]}$. Pathways transduced by the activation of TNF receptor-1 are linked to the induced expression of COX-2 ${ }^{[23]}$. Furthermore, these cytokines may stimulate the expression of 

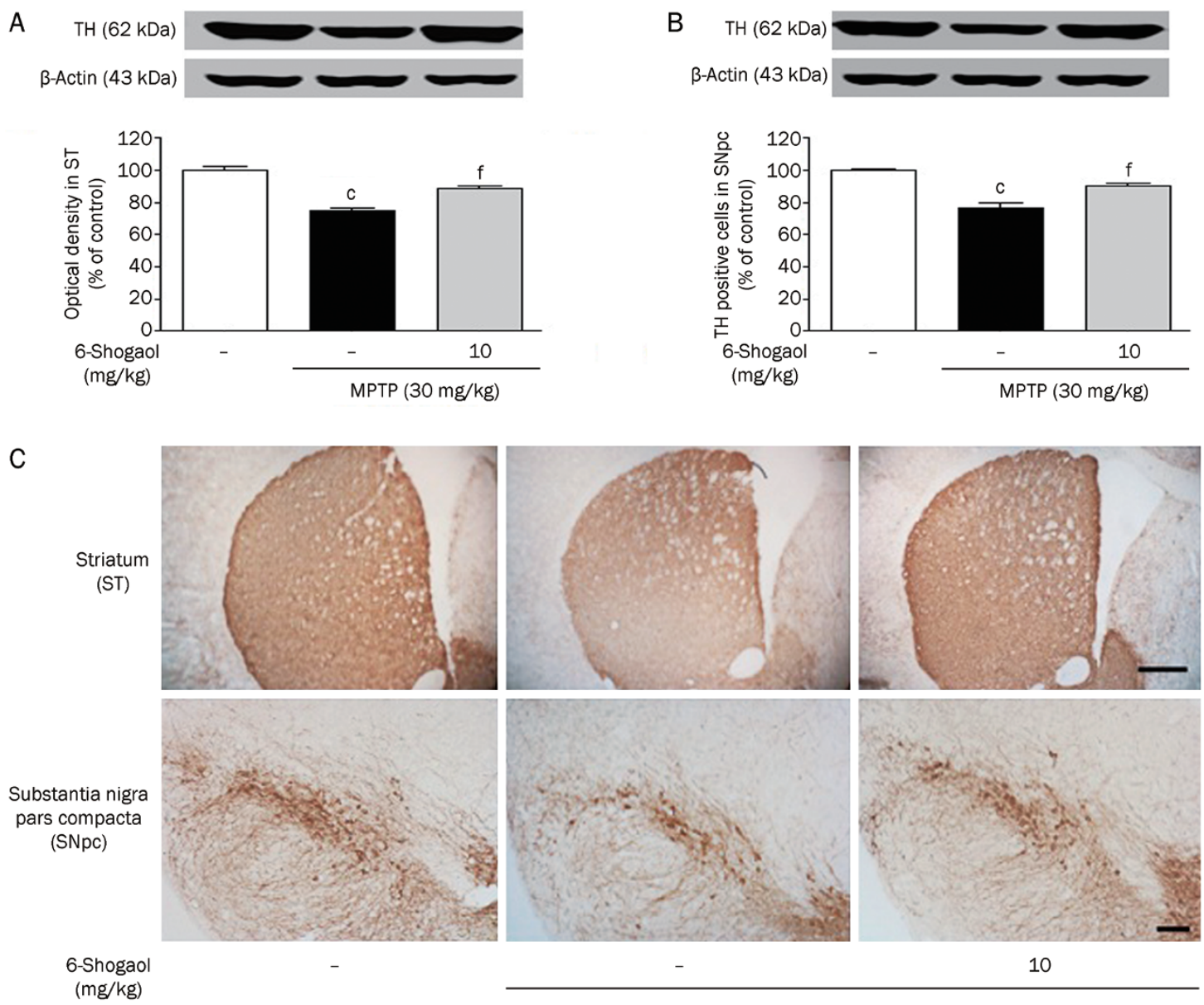

MPTP (30 mg/kg)

Figure 5. Protective effect of 6-shogaol against MPTP-induced dopaminergic neuron damage in a mouse PD model. Seven days after 6-shogaol and/or MPTP treatment, tyrosine hydroxylase (TH) tyrosine hydroxylase was detected immunohistochemically. Dopaminergic cells were quantified by counting the number of TH-immunoreactive cells in the ST(A) and by measuring the optical intensity in the SNpc(B). Representative photomicrographs are shown (C). Scale bar $=200 \mu \mathrm{m}$. Values are indicated as mean \pm SEM. ${ }^{\mathrm{C}} P<0.01$ compared with the control group. ${ }^{\mathrm{f}} P<0.01$ compared with the MPTP-only treated group.

iNOS in microglial cells via the expression and activation of the low affinity receptor of immunoglobulin $\mathrm{E}^{[24]}$. This process may lead to the production of toxic amounts of NO free radicals, which in turn could potentiate the expression and release of TNF-a by adjacent microglial cells and thereby further amplify the inflammatory reaction ${ }^{[15,25]}$. The accumulation of these factors is thought to contribute to neuronal damage, particularly in $\mathrm{PD}^{[26,27]}$. In the present study, 6-shogaol significantly inhibited $\mathrm{MPP}^{+}$-or MPTP-induced microglial activation and the downstream proinflammatory factors, such as TNF-a, NO, iNOS, and COX-2. Moreover, previous studies reported that 6-shogaol inhibited release of $\mathrm{NO}$ and the expression of iNOS induced by lipopolysaccharide. Also, 6-shogaol exerted its anti-inflammatory effects by inhibiting the production of prostaglandin $\mathrm{E}$ and proinflammatory cytokines, such as interleukin- $1 \beta$ and TNF- $\alpha$, and by downregulating COX-2, p38 mitogen-activated protein kinase, and nuclear factor kappa B expression $^{[12]}$.

The present study demonstrated that 6-shogaol, a pungent compound isolated from ginger, protects dopaminergic neurons against $\mathrm{MPP}^{+}$- and MPTP-induced neurotoxicity by inhibiting inflammatory pathways. Natural pungent compounds from plants have been suggested to play a role in protection via antioxidant, anti-inflammatory, and anti-carcinogenic $\operatorname{activities}^{[28,29]}$. For example, various experimental and clinical studies have shown that capsaicin and piperine isolated from pepper fruits, paradol isolated from Amomum melegueta Roscoe, and allicin isolated from garlic exhibit the most potent neuroprotective and anti-inflammatory effects identified for plant compounds to date ${ }^{[28,30,31]}$. Thus, 6-shogaol may contribute to a protective action against neuroinflammation in PD, although this remains to be studied further.

In summary, this study report a neuroprotective effect of 6-shogaol in a PD model: 6-shogaol protected dopaminergic cells against $\mathrm{MPP}^{+}$- and MPTP-induced neurotoxicity via the inhibition of neuroinflammatory responses of microglia in a PD model. 

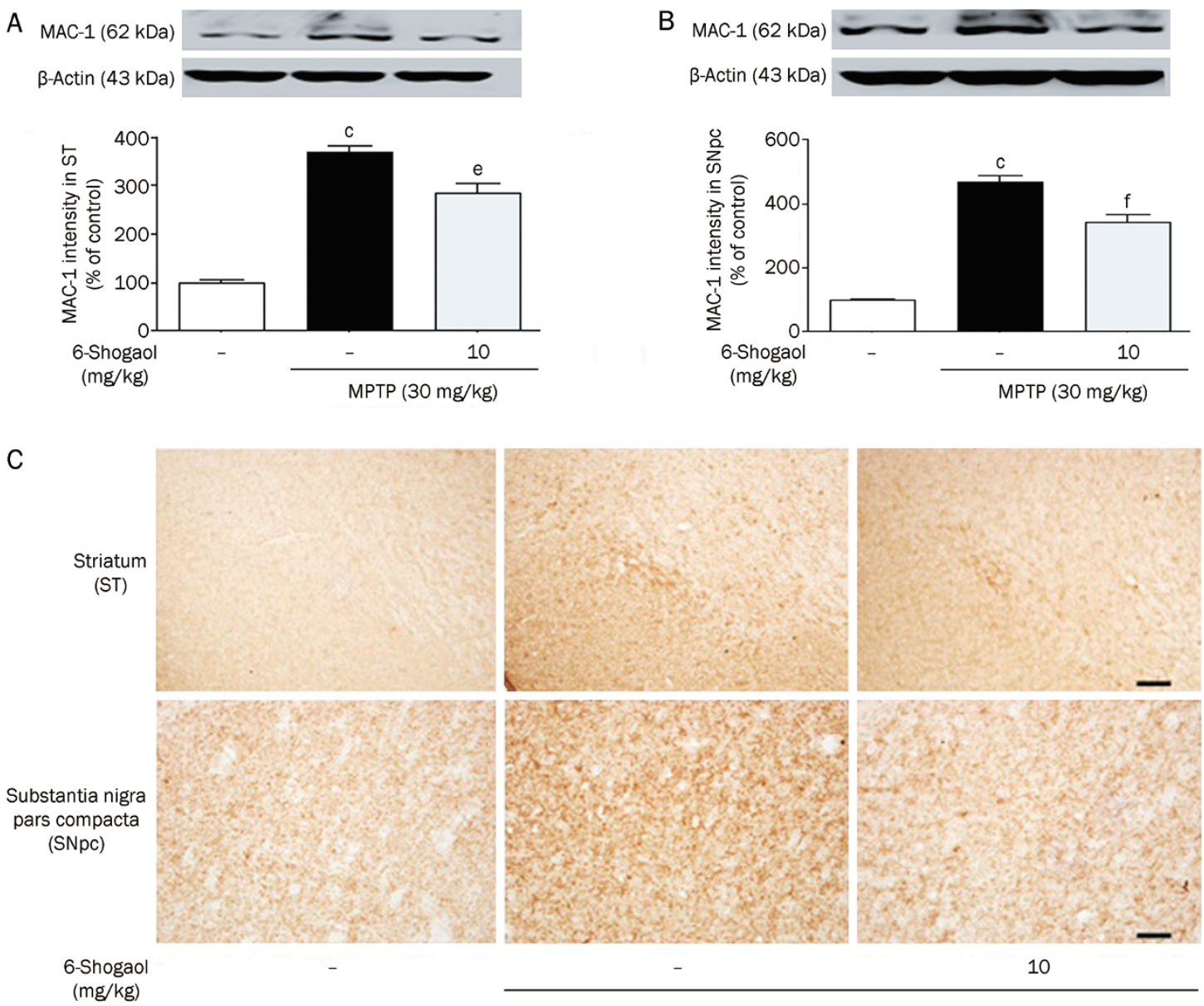

MPTP (30 mg/kg)

Figure 6. Inhibitory effect of 6-shogaol on MPTP-induced microglial activation in a mouse PD model. One day after the last MPTP treatment, MAC-1 was detected immunohistochemically. MAC-1-immunoreactive cells were quantified by counting the number of MAC-1-IR cells and measuring the optical intensity in the $\mathrm{ST}(\mathrm{A})$ and $\mathrm{SNpc}(\mathrm{B})$. Representative photomicrographs are shown $(\mathrm{C})$. Scale bar $=200 \mu \mathrm{m}$. Values are indicated as mean $\pm \mathrm{SEM}$. ${ }^{\mathrm{c}} P<0.01$ compared with the control group. ${ }^{\mathrm{e}} P<0.05,{ }^{\mathrm{f}} \mathrm{P}<0.01$ compared with the MPTP-only treated group.

\section{Acknowledgements}

This research was supported by Bio-industry Technology Development Program, Ministry for Food, Agriculture, Forestry and Fisheries, Republic of Korea (112136).

\section{Author contribution}

Myung Sook $\mathrm{OH}$ and Sun Yeou KIM designed research; Sang Keun HA, Mi Sun JU, Yongkon PARK, Hyo Geun KIM, and Gunhyuk PARK performed research; Gunhyuk PARK wrote the paper.

\section{References}

1 Ransohoff RM, Perry VH. Microglial physiology: unique stimuli, specialized responses. Annu Rev Immunol 2009; 27: 119-45.

2 Perry VH, Nicoll JAR, Holmes C. Microglia in neurodegenerative disease. Nat Rev Neurol 2010; 6: 193-201.

3 Przedborski S. Neuroinflammation and Parkinson's disease. Handb Clin Neurol 2007; 83: 535-51.

4 Schwartz M, Shechter R. Systemic inflammatory cells fight off neurodegenerative disease. Nat Rev Neurol 2010; 6: 405-10.

5 Zick SM, Djuric Z, Ruffin MT, Litzinger AJ, Normolle DP, Alrawi S, et al.
Pharmacokinetics of 6-gingerol, 8-gingerol, 10-gingerol, and 6-shogaol and conjugate metabolites in healthy human subjects. Cancer Epidemiol Biomarkers Prev 2008; 17: 1930-6.

6 Chen CC, Kuo MC, Ho CT. High performance liquid chromatographic determination of pungent gingerol compounds of ginger (Zingiber officinale Roscoe). J Food Sci 1986; 51: 1364-65.

7 Asami A, Shimada T, Mizuhara Y, Asano T, Takeda S, Aburada T, et al. Pharmacokinetics of 6-shogaol, a pungent ingredient of Zingiber officinale Roscoe (Part I). J Nat Med 2010; 64: 281-7.

8 Kyung KS, Gon JH, Geun KY, Sup JJ, Suk WJ, Ho KJ. 6-Shogaol, a natural product, reduces cell death and restores motor function in rat spinal cord injury. Eur J Neurosci 2006; 24: 1042-52.

9 Pertz HH, Lehmann J, Roth-Ehrang R, Elz S. Effects of ginger constituents on the gastrointestinal tract: role of cholinergic M3 and serotonergic 5-HT3 and 5-HT4 receptors. Planta Med 2011; 77: 973-8.

10 Shim S, Kim S, Kwon YB, Kwon J. Protection by 6-shogaol against lipopolysaccharide-induced toxicity in murine astrocytes is related to production of brain-derived neurotrophic factor. Food Chem Toxicol 2011; 50: 597-602.

11 Shim S, Kwon J. Effects of 6-shogaol on cholinergic signaling in HT22 cells following neuronal damage induced by hydrogen peroxide. Food 
A

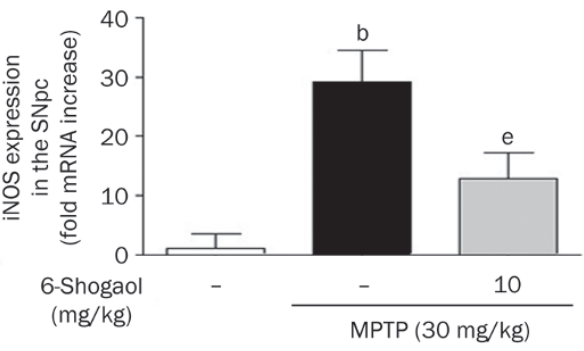

C

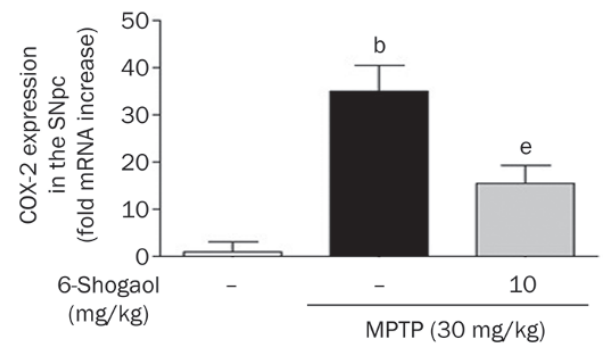

E
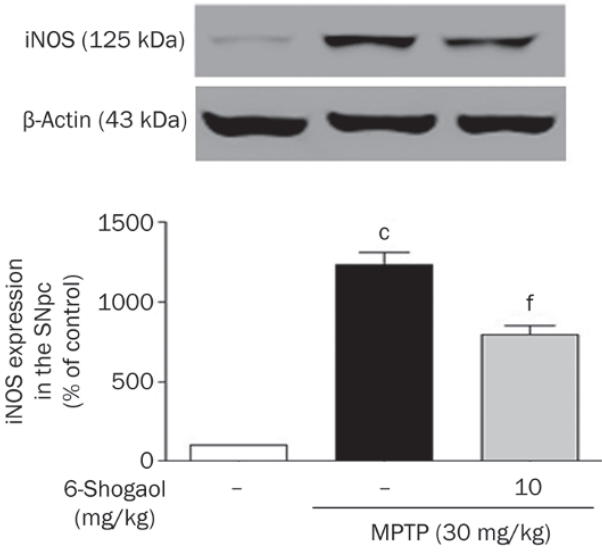

G
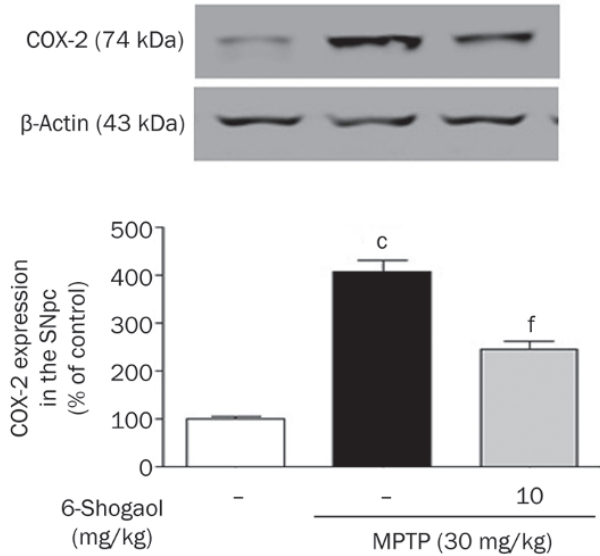

B

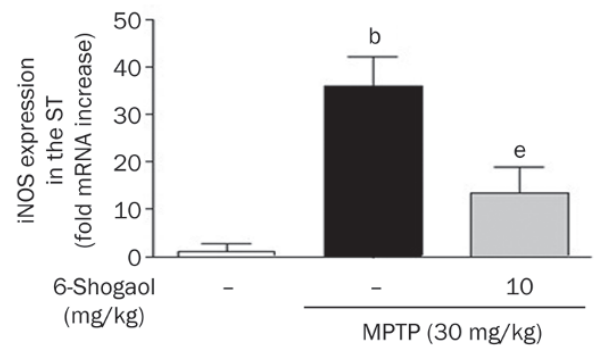

D

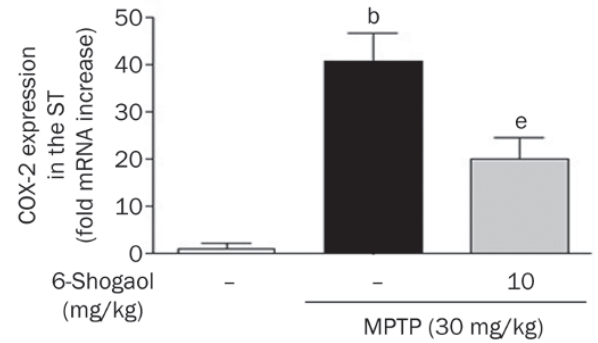

F
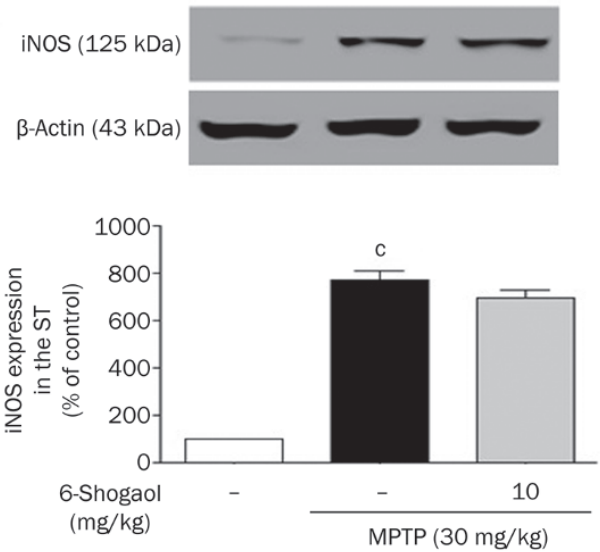

$\mathrm{H}$
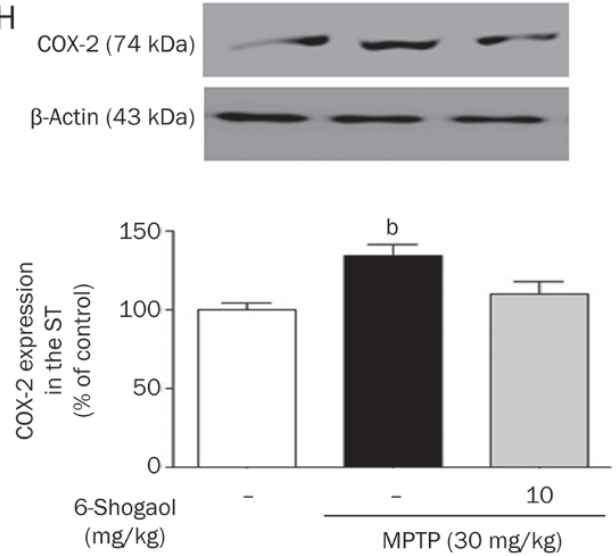

Figure 7. Inhibitory effects of 6-shogaol on MPTP-induced iNOS and COX-2 overexpression in the SNpc and ST. One day after the last MPTP treatment, brain tissues were dissociated and real-time RT-PCR and Western blot were used to determine the expression of iNOS in the SNpc (A and E) and in the ST (B and $\mathrm{F}$ ), and COX-2 in the SNpc (C and $\mathrm{G})$ and in the ST (D and $\mathrm{H})$. Values are indicated as mean \pm SEM. ${ }^{\mathrm{b}} P<0.05$, ${ }^{\mathrm{c}} P<0.01$ compared with the control group. ${ }^{\mathrm{e}} P<0.05,{ }^{\mathrm{f}} \mathrm{P}<0.01$ compared with the MPTP-only treated group. 
Chem Toxicol 2012; 50: 1454-9.

12 Ha SK, Moon E, Ju MS, Kim DH, Ryu JH, Oh MS, et al. 6-Shogaol, a ginger product, modulates neuroinflammation: A new approach to neuroprotection. Neuropharmacology 2012; 63: 211-23.

13 Tieu K. A guide to neurotoxic animal models of Parkinson's disease. Cold Spring Harbor Perspect Med 2011; 1: a009316.

14 Przedborski S, Vila M. MPTP: a review of its mechanisms of neurotoxicity. Clin Neurosci Res 2001; 1: 407-18.

15 Shaw CA, Höglinger GU. Neurodegenerative diseases: neurotoxins as sufficient etiologic agents? Neuromolecular Med 2008; 10: 1-9.

16 Depboylu C, Schorlemmer K, Klietz M, Oertel WH, Weihe E, Höglinger $\mathrm{GU}$, et al. Upregulation of microglial C1q expression has no effects on nigrostriatal dopaminergic injury in the MPTP mouse model of Parkinson disease. J Neuroimmunol 2011; 236: 39-46.

17 Ros-Bernal F, Hunot S, Herrero MT, Parnadeau S, Corvol JC, Lu L, et al. Microglial glucocorticoid receptors play a pivotal role in regulating dopaminergic neurodegeneration in parkinsonism. Proc Nati Acad Sci U S A 2011; 108: 6632-7.

18 Liu J, Wang MW, Gu P, Ma QY, Wang YY, Geng Y, et al. Microglial activation and age-related dopaminergic neurodegeneration in MPTPtreated SAMP8 mice. Brain Res 2010; 1345: 213-20.

19 Lee KW, Zhao X, Im JY, Grosso H, Jang WH, Chan TW, et al. Apoptosis signal-regulating kinase 1 mediates MPTP toxicity and regulates glial activation. PLoS One 2012; 7: e29935.

20 Phani S, Loike JD, Przedborski S. Neurodegeneration and inflammation in Parkinson's disease. Parkinsonism Relat Disord 2012; 18 : S207-9.

21 Przedborski S. Inflammation and Parkinson's disease pathogenesis. Mov Disord 2010; 25: S55-7.
22 Khasnavis S, Jana A, Roy A, Mazumder M, Bhushan B, Wood T, et al. Suppression of nuclear factor-kB activation and inflammation in microglia by physically modified saline. J Biol Chem 2012; 287: 29529-42.

23 Glass CK, Saijo K, Winner B, Marchetto MC, Gage FH. Mechanisms underlying inflammation in neurodegeneration. Cell 2010; 140: 91834.

24 Amor S, Puentes F, Baker D, Van Der Valk P. Inflammation in neurodegenerative diseases. Immunology 2010; 129: 154-69.

25 Whitton P. Inflammation as a causative factor in the aetiology of Parkinson's disease. Br J pharmacol 2007; 150: 963-76.

26 Tansey MG, Goldberg MS. Neuroinflammation in Parkinson's disease: its role in neuronal death and implications for therapeutic intervention. Neurobiol Dis 2010; 37: 510-8.

27 Lim HJ, Li H, Kim JY. Quercetin derivatives from Siegesbeckia glabrescens inhibit the expression of COX-2 through the suppression of NF-kB activation in microglia. Biomal Ther 2011; 19: 27-32.

28 McNamara FN, Randall A, Gunthorpe MJ. Effects of piperine, the pungent component of black pepper, at the human vanilloid receptor (TRPV1). Br J pharmacol 2005; 144: 781-90.

29 Patacchini R, Maggi CA, Meli A. Capsaicin-like activity of some natural pungent substances on peripheral endings of visceral primary afferents. Naunyn Schmiedebergs Arch Pharmacol 1990; 342: 72-7.

30 Waggas AM. Neuroprotective evaluation of extract of ginger (Zingiber officinale) root in monosodium glutamate-induced toxicity in different brain areas male albino rats. Pak J Biol Sci 2009; 12: 201-12.

31 Subramanian U, Poongavanam S, Vanisree A. Studies on the neuroprotective role of Piper longum in $\mathrm{C} 6$ glioma induced rats. Invest New Drugs 2010; 28: 615-23. 